\title{
A Survey on Medium Access Control (MAC) for Clustering Wireless Sensor Network
}

\author{
$1^{\text {st }}$ A. Anhar \\ Dept. of Electrical and Electronic Engg. \\ Brunel University \\ London, UK \\ Anhar.Anhar@brunel.ac.uk
}

\author{
$2^{\text {nd }}$ R. Nilavalan \\ Dept. of Electrical and Electronic Engg. \\ Brunel University \\ London, UK \\ Rajagopal.Nilavalan@brunel.ac.uk
}

\author{
$3^{\text {rd }}$ Febrizal Ujang \\ Dept. of Electrical Engg. \\ University of Riau \\ Pekanbaru, Indonesia \\ febrizal@eng.unri.ac.id
}

\begin{abstract}
Wireless Sensor Network (WSN) has gained enormous popularity since it is a base of an Internet of Things and applied in many areas of application. However, this network suffers an energy constraint issue which has to be tackled. Medium Access Control (MAC) manages the radio communication of transmitter and receiver, which is the highest energy consumed of sensor nodes. Therefore, many researchers have been proposed new MAC protocols to overcome this limitation. This paper explores and compares several MAC protocols for clustering WSN. Clustering network has a different characteristic since it classifies nodes as Cluster Heads and Cluster Members. This leads to a unique approach in developing MAC to enhance the network's performance.
\end{abstract}

Index Terms-Wireless Sensor Network, Medium Access Control, Clustering Network, Energy Constraint.

\section{INTRODUCTION}

Nowadays it becomes easy to monitor environmental condition surround us, thanks to Wireless Sensor Network (WSN) combining sensors and wireless technologies. It is a low-cost technology and easy to deploy in a broad area of communication [1] ranging from monitoring environment to smart application. WSN has the ability to sense, process, and transmit data using a wireless medium such as radio frequency wave, infrared, Bluetooth, or any other wireless media.

Generally, WSN consists of tiny nodes called motes and one or multiple nodes named as a sink or a base station (BS) [2]. As shown in fig.1, the node hardware is composed of a detecting module, a data processing module, a transceiver module, and a power module [3]-[5]. Data in sensor nodes are collected from a sensor unit. These data are an analogue signal and converted to a digital signal using analogue to digital converter (ADC). The digital data are processed in microprocessors or microcontrollers as a processing module. Following this, data are transmitted to the BS via a transceiver unit which consumes the highest energy. All units need an energy source supplied by a power module.

Despite advantages, WSN faces some issues such as a low end to end delay, throughput, and scalability. These can be different for a particular application. For instance, in the emergency application, data should be delivered to the sink with a minimum end to end delay. However, the main issue for many applications is energy constraint since tiny mote is supplied by limited batteries. Commonly battery in a sensor node is AA, AAA, or Li-ion [5]. Recharging battery in some applications is impossible.

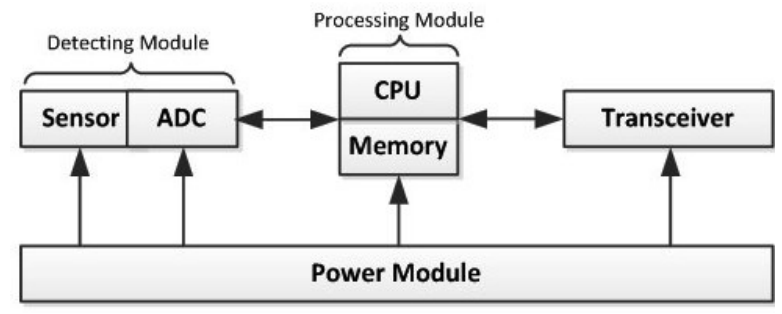

Fig. 1. The elements of a Sensor Node.

Many researchers have developed approaches to tackle WSN's issues ranging from a physical layer to a network layer. Medium Access Layer (MAC), a sublayer of Link Layer (LL), has much impact in achieving better performance in WSN. MAC protocol directly controls when a node transmits or receives data. This process influences energy consumed in a sensor node. Moreover, collision packets which contribute to the network throughput and end to end delay can be minimised by an effective MAC.

Due to a significant impact of MAC on the network performance, several attempts have been proposed. Every proposed MAC has different features and methods in tackling WSN's limitation. In order to explore and understand in depth all the proposed approaches, recently, many researchers have shown an increased interest in reviewing the proposed MAC including drawbacks and advantages. Authors commonly focused on mechanisms to access the shared medium, quality of service, and network traffic. However, no research has been found that surveyed MAC protocol considering the network topology. A certain topology such as clustering has different characteristic and is one of the algorithms to minimise the energy consumed [6]. This work explores MAC on clustering WSN. Clustering WSN grouping sensor nodes in a cluster are one of the network topologies that have been applied in many applications, especially in monitoring application. Furthermore, the work analyses advantages and disadvantages and compares features of clustering MAC.

The rest of the paper is organised as follows: Section 2 provides the brief review of the related work. Classification of MAC is explained in section 3. In part 4, sources of issues of MAC are presented. The detail and comparison of MAC for clustering WSN is in section 5. Finally, the paper is concluded in section 6 . 


\section{RELATED WORKS}

Many papers are reviewing MAC layer in WSN. In the early stage, the survey's papers generally explored MAC protocol without classification. Work on [7] is the first review paper on MAC. Some works such as [8]-[11] are other papers that do not have a taxonomy in reviewing MAC protocol; therefore, it is difficult to characterise or differentiate every proposed MAC.

To give more understanding in state of the art MAC protocol, many surveys have grouped MAC along with its characteristic. Authors in [12], [13] provided the classification of MAC based on how they access the shared medium: reservation-based and contention-based MAC. Authors in [14] introduced a sophisticated taxonomy of MAC according to energy efficiency and data delivery. MAC in this work was classified into four categories: synchronous, asynchronous, frame-slotted, and multi-channel.

Since the central issue in WSN is energy constraint, there have been many proposed approach from a physical layer to network layer focusing on solving this issue. As a part of the link layer, MAC can be designed to achieve energy efficiency. Therefore, several reviews works on MAC have explored the effectiveness of MAC in term of energy. The first attempt is the authors in [15]. At the beginning of the paper, the authors presented the energy management in WSN as a primary concept to understand sources of energy exhausted and how to minimise them. Some energy mechanism techniques such as duty cycle, energy-efficient scheduling, scheduled rendezvous, on-demand wake-up scheme, energyefficient through the directed antenna, and clustering are discussed in more detail. The paper also classified MAC protocol as a centralised protocol, distributed protocol, and a hybrid protocol. In [16], authors only divided MAC into synchronous and asynchronous while work in [17] grouped MAC as contention-based protocol and hybrid-based protocol. Another taxonomy according to the design method was introduced by [18], and MAC in this approach was categorised into two main groups: single layer and crosslayer.

As sensor and communication technology have been developing fast, a real-time and multimedia is a new paradigm of WSN application. This application requires not only energy efficient but also reliability in delivering packets and low end-to-end delay. Many researchers shifted their research attention to this application. Accordingly, works on reviewing MAC protocol investigated this objective such as [19]-[22]. The sampling rate and the density of multimedia sensor as parameters on multimedia data, for example, were analysed in depth with the relation of energy efficiency. Furthermore, the authors investigated the advantages and disadvantages of the proposed MAC and explored their suitability for the realtime application.

To reduce the amount of energy consumed, MAC protocols in recent years have introduced the idea of wake-up radio hardware. This device is a small component that added in the transceiver of the sensor node to listen to the shared medium while the node is in sleep mode. It can react to the external event and wake up the node. Regarding this technique, Djroun et al. [23] reviewed the state-of-the-art of
MAC using the wake-up radio system. Authors divided the wake-up radio into passive and active, and explore in detail both of the components. On the other hand, Sherazi et al. [24] surveyed MAC on Energy Harvesting WSN (EHWSN). It is a promising technology, and many researchers and industries have been investigated its possible to enhance the network lifetime combining with other protocols such as MAC. This manuscript comprehensively focused on: fundamental techniques, evaluation approaches, and key performance indicators.

Arain et al. [2] reviewed 30 surveys papers regarding on MAC in WSN. This work classified the survey's article based on the subject-wise, which are: general, taxonomy, quality of service, performance, eccentric, and energy-efficiency. Besides, the authors analysed the coverage of the survey's paper and calculated the impact factor of every proposed MAC.

\section{NETWORK TOPOLOGY OF WSN}

Generally, a topology of WSN can be classified into two categories: flat topology and cluster topology [25]-[27]. In flat topology, every sensor node has the same function in the network. As shown in fig.2, data from a node can be sent directly to the BS or other nodes which are close to the BS. For later technique, it is known as multi-hop communication. In choosing the next node, the node considers many factors, such as distance, remaining energy, link state, and traffic of the node. In contrast, clustering WSN classifies nodes as Cluster Head $(\mathrm{CH})$ and Cluster Member (CM). A CH can control the access to the shared medium while a $\mathrm{CM}$ is a sensor node which has to follow the instruction from $\mathrm{CH}$ regarding transmitting data. Fig.3 illustrates the transmission process in clustering topology.

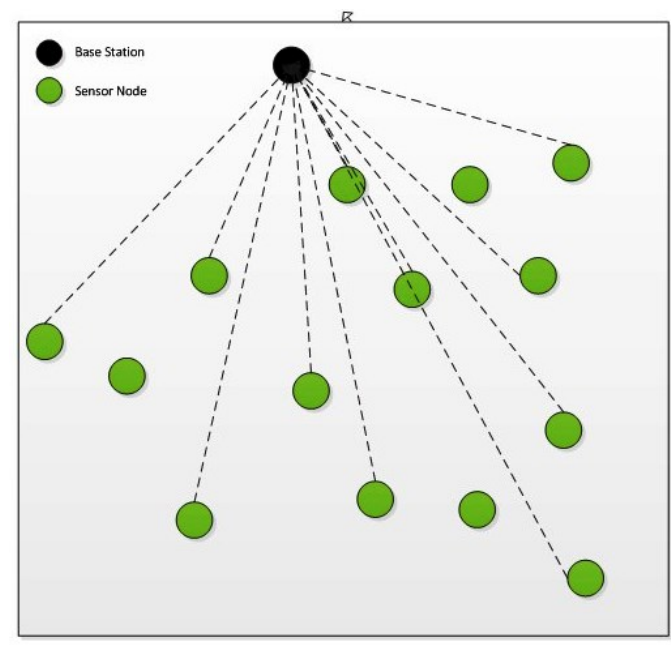

Fig. 2. A Flat Topology of WSN

Since no coordinator or $\mathrm{CH}$ in flat WSN, Carrier Sense Multiple Access with Collision Avoidance (CSMA-CA) commonly is adopted as a fundamental algorithm to access the wireless medium. If the number of traffic is low, this approach has a small collision. As a result, the average end-to-end delay and energy consumed are low. This mechanism is suitable for detection and non-monitoring application. On the other hand, most clustering WSN apply TDMA based 
MAC in accessing the medium because there exist $\mathrm{CHs}$ that control the communication activities of nodes. For monitoring application, a CM senses environment continuously and sends it to the BS. By clustering sensor nodes and applying TDMA scheme, data from CM are transmitted in a fixed time slot, then aggregated in $\mathrm{CH}$ before sending it to the BS. This has to be done to avoid data collision and reduce the number of data forwarding to the BS.

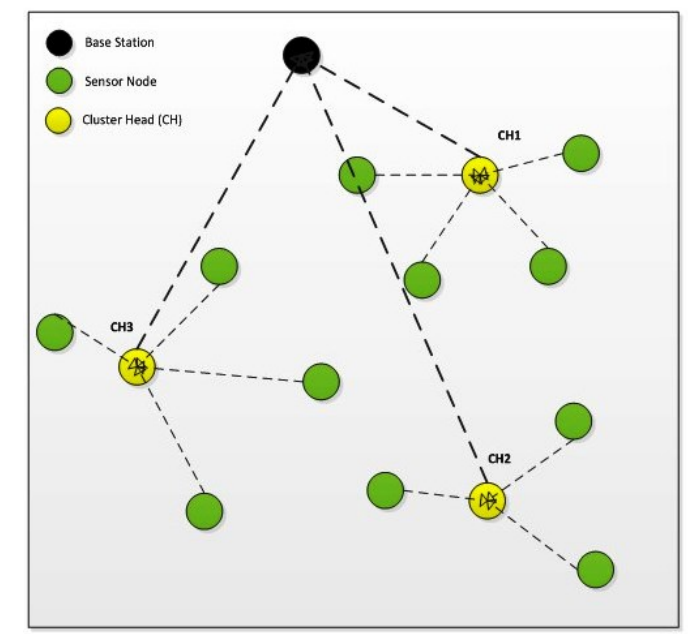

Fig. 3. A Clustering Topology of WSN

\section{MAC IN WSN}

As was pointed out in the introduction of this paper, Medium Access Control (MAC) protocol always plays a significant role in the design of WSNs as considerable energy consumption is due to the sensing, reception, and transmission process [24]. In this section, we list the performance metrics of an effective MAC and following this, we analyse factors affecting those metrics.

Generally, four metrics are used to measure the effectiveness of the proposed MAC, as listed below [6], [28], [29].

- Energy efficiency: It is the total energy consumed divided by total packets in the network.

- Latency: This is the time when the packet leaves the sender until it arrives at the destination.

- Throughput: It is the total number of packet arrived in destination divided by time.

- Scalability: It is the ability of network in handling a large number of nodes.

After knowing these factors, we explore the significant elements influencing the trade-off of performance metrics. These elements are:

- Collision: When two nodes have data to be sent at the same time, a collision occurs in the wireless network. These collision packets are wasted packets, and nodes have to wait to retransmit these packets. Therefore, collision consumes nodes energy and adds an extra delay to packets.

- Overhearing: When a node is in active mode, it will receive all messages within its transmission range although these messages are not addressed to it.
- Idle listening: It is a mechanism to listen continuously to wireless medium although there is no transmission on the channel.

- Overhead: It is controlling messages and additional bits in the protocol header. The more overhead packets in the network, the more energy are consumed.

\section{COMPARING MAC FOR CLUSTERING WSN}

After discussing performance metrics and factors that influencing it, this section explains several MAC for clustering WSN and compares their performance regarding energy consumed, latency, throughput, and scalability.

\section{A. Energy Time Division Multiple Access (E-TDMA)}

Heinzelman introduced E-TDMA in [30]. It is a TDMA MAC where every node as a member of cluster head has a fixed duration in transmission data. Before sending data, a $\mathrm{CM}$ has to choose a $\mathrm{CH}$ based on the distance from the $\mathrm{CM}$ to the $\mathrm{CH}$. Following this, the $\mathrm{CH}$ sends TDMA frame containing a sequence of transmitting data from CMs. The $\mathrm{CM}$ which is close to the $\mathrm{CH}$ is the first one transmitting data with the fixed duration. Fig. 4 illustrates the TDMA frame of this approach.

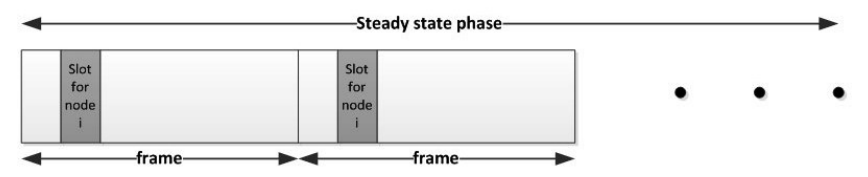

Fig. 4. A Frame of E-TDMA

E-TDMA assumes that every CM always has data to be sent to $\mathrm{CH}$. If a $\mathrm{CM}$ dies because of energy or any other factors, there will be an empty slot in the TDMA frame. The frame cannot be replaced by others until the next round when a new $\mathrm{CH}$ is chosen and send a new TDMA frame. Furthermore, when the number of $\mathrm{CM}$ is small, every $\mathrm{CM}$ send data more frequently. As a result, these CMs suffer the high energy loss. Another drawback is that E-TDMA does not support multi-hop communication which is needed for long distance transmission. MAC for multi-hop communication is different with single-hop since $\mathrm{CHs}$ in multi-hop serve not only as a $\mathrm{CH}$ but also as an intermediate node forwarding data from other CHs. The link reliability should be high to avoid losing any data in the network.

\section{B. Slot Stealing Medium Access Control}

Authors in [31] introduced Slot Stealing Medium Access Control (SS-MAC) for star or clustering network. Nodes in WSN are classified into two clusters: nodes with emergency and nonemergency traffic. The following traffic is used for monitoring, and the first one is for critical data. The number of the node transmitting emergency traffic depends on the application and sets up precisely to detect the emergency event upon the network deployment.

Channel access mechanism for transmitting monitoring data follows a TDMA scheme. Every node has a fixed time slot to send data to the $\mathrm{CH}$ or a controller. When there is no emergency traffic, time slots for nodes with emergency traffic remain null. An Emergency Indication Subslot (EIS) 
with a duration TEIS is placed between two consecutive time slots. It will be used in an emergency situation and signs for a busy channel. Nodes finding the busy signal will postpone its transmission until the next time slot available. If a node has emergency data, firstly it sends EIS to enable slot stealing from normal nodes. Following this, it transmits a Reservation Request Packet (RRP) to ensure channel allocation for its transmission. The size of RRP packet is small enough to guarantee that it gets the highest priority in the network. If there are two or more nodes with the same priority, the controller applies the First-Come-First-Served (FCFS) method to schedule the transmission of nodes.

SS-MAC initially chooses some nodes as the high priority nodes. This selection must be made before the deployment of sensor nodes. For some emergency application including forest fire monitoring, nodes with emergency traffics cannot be predetermined due to the random location of nodes transmitting these data. As a result, SS-MAC is not suitable for monitoring forest fire or any uncertain interest. SS-MAC assumes that every node can reach the controller or $\mathrm{CH}$ directly without considering the distance. This direct communication is not applicable if the distance between them is far. Furthermore, as the distance increases, the energy consumed grows up significantly. Another drawback is the formation of clustering. This approach only groups nodes into two clusters based on the traffic. In many pieces of research, grouping should be dealt with many factors including distance, remaining energy, QoS, and the position of BS [32], [33]. These parameters can enhance the network performance.

\section{Hierarchical Energy-Efficient MAC}

The concept of TDMA in MAC is also introduced by Sharma et al. in [34] and called Hierarchical Energy-Efficient MAC (HEEMAC). This approach has the same algorithm as E-TDMA and is designed for clustering network. In intra communication (transmission between $\mathrm{CM}$ and $\mathrm{CH}$ ), CM transmits its data according to the TDMA frame time which is advertised by $\mathrm{CH}$. This approach applies Hard Threshold (HT) and Soft Threshold as in [35]. A sensor node senses the environment regularly and sends to the $\mathrm{CH}$ if the detected value achieves HT. The next transmission will exist if the variation of sensed value exceeds ST. Multi-hops communication is applied in this approach to support longdistance transmission. Data from $\mathrm{CH}$ are sent to the next $\mathrm{CH}$ which is close to the BS.

Adoption of HT and ST lead to energy efficiency since it can reduce the number of transmission data to the BS. However, only a sensed value which is higher than HT will be sent to the BS. This method is not appropriate for monitoring application due to some data from sensor nodes are not assigned to $\mathrm{CH}$. In addition, a TDMA frame which is transmitted by $\mathrm{CH}$ is not usable because a sensor node does not have a sensed data continuously. When a node has data to be sent, it has to wait for its TDMA time slot. This waiting mechanism causes an extra delay in transmitting data. In addition, there is no priority mechanism to send an emergency data to the BS. Hence this approach is not suitable for monitoring emergency service.

\section{Bit-map-assisted Energy-Efficient(BMA) MAC}

Bit-map-assisted Energy-Efficient (BMA) MAC is a novel MAC for clustering network based on a TDMA approach [36]. It only sends significant data to the $\mathrm{CH}$ to minimise the energy consumed. This characteristic is suitable for an event-driven application. There are two phases in BMA: setup phase, and steady-state phase. Choosing $\mathrm{CHs}$ which is accomplished in the set-up phase follows the mechanism of Low Energy Adaptive Clustering (LEACH) protocol [30]. On the other hand, the algorithm in steady-state phase is complicated since there are $\mathrm{k}$ sessions in the steady-state phase and every session contains a contention period, a data transmission period, and an idle period. At the beginning of contention period, a $\mathrm{CH}$ broadcast a TDMA frame, and every $\mathrm{CM}$ sends a 1-bit control message to inform the $\mathrm{CH}$ that it has data to be sent or not. If a CM has data, it enters a transmission period and should transmit a control message. This node is called as a source node. Otherwise, this slot remains empty. Based on these control messages, the $\mathrm{CH}$ creates a new TDMA slot for the source node. If nodes do not have data, it turns off its communication device and enters an idle period.

A source node in BMA has to wait for the contention period before transmitting data. This leads an extra delay for a node. Furthermore, unlike in E-TDMA which has one advertisement, there exists two TDMA advertisements from $\mathrm{CH}$ : the first advertisement for contention period, and the second advertisement for transmission period. These advertisement delays bring an extra delay for a node. For long-distance communication, there is no multi-hop communication since this approach assumes that every $\mathrm{CH}$ can communicate directly to the BS without considering the distance. As a result, if the position of the BS is far away from the $\mathrm{CH}$, the amount of energy expenditure will be high.

\section{E. Comparing MAC for clustering WSN}

After discussing several MAC protocols for clustering WSN, this section analysis comparing the performance of clustering MACs. As shown in table I, there are four performance metrics and two mechanisms related to MAC protocol. Scheduling is an approach in transmitting a packet to the destination. It is classified into three parts: dynamic, static, and mixed approach. In the dynamic approach, a sender sends messages in an uncertain time slot while in the static approach, a sender transmits messages in a fixed time slot. A mixed approach combines dynamic and fixed approaches. Clustering formation in WSN is grouped into centralised, distributed, and mixed clustering. The BS chooses $\mathrm{CHs}$ in centralised clustering meanwhile nodes itself selects their $\mathrm{CH}$ in distributed clustering. Moreover, mixed clustering is a combination of centralised and distributed clustering.

Some convicting comments can be drawn from the table above. Firstly, HEE-MAC outperforms others in term of energy efficiency, latency, and scalability due to the threshold and multi-hop communication approach. Unlike E-TDMA and SS-MAC having high throughput, the number of packets received in HEE-MAC is low since only sensed data reaching hard and soft threshold can be transmitted to the destination. On the contrary, E-TDMA as the base of clustering MAC has a low performance regarding energy consumed, end-to-end 
TABLE I

COMPARISON OF MAC PROTOCOLS

\begin{tabular}{|c|c|c|c|c|}
\hline Protocol & E-TDMA & SS-MAC & HEE-MAC & BMA MAC \\
\hline $\begin{array}{c}\text { Energy } \\
\text { Efficiency }\end{array}$ & Low & Normal & High & High \\
\hline Latency & Low & High & High & High \\
\hline Throughput & High & High & Low & Low \\
\hline Scalability & Low & Low & High & Low \\
\hline Scheduling & Fixed & Fixed & Fixed & Fixed \\
\hline $\begin{array}{c}\text { Clustering } \\
\text { Formation }\end{array}$ & Distributed & Centralised & Distributed & Distributed \\
\hline
\end{tabular}

delay, and the scalability. This MAC has a high throughput because all data from $\mathrm{CMs}$ are sent to the $\mathrm{CH}$ regularly.

\section{ACKNOWLEDGMENT}

The authors wish to thank the Directorate General of Resources for Science, Technology and Higher Education, the Ministry of Research, Technology and Higher Education of the Republic of Indonesia for providing A. Anhar a PhD scholarship to pursue his study at Brunel University London, UK.

\section{REFERENCES}

[1] Y. Liu, K. Ota, K. Zhang, M. Ma, N. Xiong, A. Liu, and J. Long, QTSAC: An Energy-Efficient MAC Protocol for Delay Minimization in Wireless Sensor Networks, IEEE Access, vol. 6, no. Part II, pp. 82738291, 2018

[2] W. M. Arain and S. Ghani, Meta-survey on medium access control surveys in wireless sensor networks, Int. J. Distrib. Sens. Networks, vol. 12, no. 8, 2016.

[3] J. N. Al-Karaki and a. E. Kamal, Routing Techniques in Wireless Sensor Networks: A Survey, IEEE Wirel. Commun., vol. 11, no. 6, pp. 628, 2004.

[4] J. Aponte-Luis, J. Gmez-Galn, F. Gmez-Bravo, M. Snchez-Raya, J. Alcina-Espigado, and P. Teixido-Rovira, An Efficient Wireless Sensor Network for Industrial Monitoring and Control, Sensors, vol. 18, no. 1, p. 182,2018

[5] M. DENER, WiSeN: A new sensor node for smart applications with wireless sensor networks, Comput. Electr. Eng., vol. 64, pp. 380394, Nov. 2017.

[6] V. Mhatre and C. Rosenberg, Design guidelines for wireless sensor networks: Communication, clustering and aggregation, Ad Hoc Networks, vol. 2, no. 1, pp. 4563, 2004

[7] I. F. Akyildiz, W. Su, Y. Sankarasubramaniam, and E. Cayirci, A survey on sensor networks, IEEE Commun. Mag., vol. 40, no. 8, pp. 102105, 2002 .

[8] I. Demirkol, C. Ersoy, and F. Alagoz, MAC protocols for wireless sensor networks: a survey, IEEE Commun. Mag., vol. 44, no. 4, pp. 115121, Apr. 2006

[9] P. Baronti, P. Pillai, V. W. C. Chook, S. Chessa, A. Gotta, and Y. F. $\mathrm{Hu}$, "Wireless sensor networks: A survey on state of the art and the 802.15.4 and ZigBee standards," Comput. Commun., vol. 30, no. 7, pp. 16551695, 2007.

[10] Y. Z. Zhao, C. Miao, M. Ma, J. B. Zhang, and C. Leung, A survey and projection on medium access control protocols for wireless sensor networks, ACM Comput. Surv., vol. 45, no. 1, pp. 137, 2012

[11] A. Verma, M. P. Singh, J. P. Singh, and P. Kumar, Survey of MAC Protocol for Wireless Sensor Networks, in 2015 Second International Conference on Advances in Computing and Communication Engineering, 2015, pp. 9297.

[12] A. Bachir, M. Dohler, T. Watteyne, and K. K. Leung, MAC Essentials for Wireless Sensor Networks, IEEE Commun. Surv. Tutorials, vol. 12 no. 2, pp. 222248, 2010.

[13] J. Kabara and M. Calle, MAC protocols used by wireless sensor networks and a general method of performance evaluation, Int. J. Distrib. Sens. Networks, vol. 2012, 2012.

[14] P. Huang, L. Xiao, S. Soltani, M. W. Mutka, and N. Xi, The evolution of MAC protocols in wireless sensor networks: A survey, IEEE Commun. Surv. Tutorials, vol. 15, no. 1, pp. 101120, 2013.
[15] M. Al Ameen, S. M. R. Islam, and K. Kwak, Energy Saving Mechanisms for MAC Protocols in Wireless Sensor Networks, Int. J. Distrib. Sens. Networks, vol. 6, no. 1, p. 163413, 2010.

[16] E. Chukwuka and K. Arshad, Energy Efficient Mac Protocols for Wireless Sensor Network: a Survey, Int. J. Sci. Eng. Res., vol. 5, no. 4, pp. 7589, 2013.

[17] K. Joice Olempia, C. Pandeeswaran, and P. Natarajan, A survey on energy efficient contention based and hybrid MAC protocols for wireless sensor networks, Indian J. Sci. Technol., vol. 9, no. 12, pp. $110,2016$.

[18] T. Maitra and S. Roy, A comparative study on popular MAC protocols for mixed Wireless Sensor Networks: From implementation viewpoint, Comput. Sci. Rev., vol. 22, pp. 107134, 2016.

[19] Z. Teng and K.-I. Kim, A Survey on Real-Time MAC Protocols in Wireless Sensor Networks, Commun. Netw., vol. 2, no. 2, pp. 104112 , 2010.

[20] M. A. Yigitel, O. D. Incel, and C. Ersoy, QoS-aware MAC protocols for wireless sensor networks: A survey, Comput. Networks, vol. 55, no. 8, pp. 19822004, Jun. 2011.

[21] M. Atto and C. Guy, "Wireless Sensor Networks: MAC Protocols and Real-Time Applications," 2012.

[22] T. AlSkaif, B. Bellalta, M. G. Zapata, and J. M. Barcelo Ordinas, Energy efficiency of MAC protocols in low data rate wireless multimedia sensor networks: A comparative study, Ad Hoc Networks, vol. 56, pp. 141157, 2017.

[23] F. Z. Djiroun and D. Djenouri, MAC Protocols With Wake-Up Radio for Wireless Sensor Networks: A Review, IEEE Commun. Surv. Tutorials, vol. 19, no. 1, pp. 587618, 2017.

[24] H. H. R. Sherazi, L. A. Grieco, and G. Boggia, A comprehensive review on energy harvesting MAC protocols in WSNs: Challenges and tradeoffs, Ad Hoc Networks, vol. 71, pp. 117134, 2018.

[25] H. Shen and G. Bai, Routing in wireless multimedia sensor networks: A survey and challenges ahead, J. Netw. Comput. Appl., vol. 71, pp. 3049, 2016.

[26] H. Karl and A. Willig, Protocols and Architectures for Wireless Sensor Networks. Chichester, UK: John Wiley \& Sons, Ltd, 2005.

[27] L. M. OLIVEIRA, L. RODRIGUES, and J. J. P. C., Wireless Sensor Networks: a Survey on Environment Monitoring, J. Commun., vol. 6, no. 2, pp. 143151, 2011.

[28] Z. Wang, M. Feng, T. Miao, W. Jiang, and J. Shen, Energy Efficient MAC Protocol for Wireless Sensor Networks: A Survey, Springer, Cham, 2017, pp. 422429.

[29] A. Asudeh, G. V. Zruba, and S. K. Das, A general model for MAC protocol selection in wireless sensor networks, Ad Hoc Networks, vol. 36, pp. 189202, 2016.

[30] W. B. Heinzelman, A. P. Chandrakasan, and H. Balakrishnan, An application-specific protocol architecture for wireless microsensor networks, IEEE Trans. Wirel. Commun., vol. 1, no. 4, pp. 660670, 2002.

[31] H. Farag, M. Gidlund, and P. Osterberg, A Delay-Bounded MAC Protocol for Mission- and Time-Critical Applications in Industrial Wireless Sensor Networks, IEEE Sens. J., vol. 18, no. 6, pp. 26072616 , 2018.

[32] K. Guleria and A. K. Verma, Comprehensive review for energy efficient hierarchical routing protocols on wireless sensor networks, Wirel. Networks, vol. 1, Mar. 2018.

[33] A. Anhar, R. Nilavalan, and M. S. Iqbal, Clustering based on the node health status in wireless sensor networks, in 2017 11th International Conference on Telecommunication Systems Services and Applications (TSSA), 2017, pp. 15

[34] R. Sharma, C. Engineering, B. S. Sohi, C. Engineering, N. Mittal, and C. Engineering, Hierarchical Energy Efficient MAC protocol for Wireless Sensor Networks, Int. J. Appl. Eng. Res., vol. 12, no. 24, pp. 1472714738, 2017

[35] M. Ghiasabadi, M. Sharifi, N. Osati, S. Beheshti, and M. Sharifnejad, TEEN: a routing protocol for enhanced efficiency in wireless sensor networks, 2008 Second Int. Conf. Futur. Gener. Commun. Netw., vol. 1, no. C, pp. 20092015, 2001

[36] J. Li and G. Y. Lazaroul, A Bit-Map-Assisted Energy-Eff icient MAC Scheme for Wireless Sensor Networks, pp. 5560, 2004. 